\title{
Particle Filtering for Nonlinear BOLD Signal Analysis
}

\author{
Leigh A. Johnston ${ }^{1,2}$, Eugene Duff ${ }^{1,3}$, and Gary F. Egan ${ }^{1}$ \\ ${ }^{1}$ Howard Florey Institute \& Centre for Neuroscience, Melbourne, Australia \\ ${ }^{2}$ Dept. of Electrical \& Electronic Engineering, University of Melbourne, Australia \\ ${ }^{3}$ Dept. of Mathematics \& Statistics, University of Melbourne, Australia \\ $\{1 . j o h n s t o n$, e.duff, g.egan\}@hfi.unimelb.edu.au
}

\begin{abstract}
Functional Magnetic Resonance imaging studies analyse sequences of brain volumes whose intensity changes predominantly reflect blood oxygenation level dependent (BOLD) effects. The most comprehensive signal model to date of the BOLD effect is formulated as a continuous-time system of nonlinear stochastic differential equations. In this paper we present a particle filtering method for the analysis of the BOLD system, and demonstrate it to be both accurate and robust in estimating the hidden physiological states including cerebral blood flow, cerebral blood volume, total deoxyhemoglobin content, and the flow inducing signal, from functional imaging data.
\end{abstract}

\section{Introduction}

Functional Magnetic Resonance (fMR) imaging methods map brain activation through the acquisition and analysis of a sequence of scanned volumes. Changes in the measured fMR signal are primarily due to the blood oxygenation level dependent (BOLD) effect, reflecting hemodynamic response to neuronal activity. The most common fMR analysis approach is based on regression of a General Linear Model, in which BOLD effect predictors are formed by convolution of a predefined hemodynamic response function with the input stimulus sequence, and the estimated predictor weights are taken as a measure of neuronal activity. The relationship between the input stimulus sequence and output BOLD signal is recognised, however, as being both subject and session dependent, and nonlinearly related to underlying physiological variables that include cerebral blood flow, cerebral blood volume, and total deoxyhemoglobin content [1, thus rendering the GLM insufficient for a detailed analysis of the system.

One of the most comprehensive nonlinear models of the BOLD signal, presented by Riera et al. 2], models the black-box system between the known input stimulus sequence and measured output BOLD signal by a continuous-time, stochastic dynamical system, in which the physiological variables form the hidden states. This model incorporates previous BOLD signal models, including the balloon-windkessel approach of [34] and the hemodynamic model linking the stimulus sequence and flow-inducing signal [5]. 
Any model is a purely descriptive tool unless methods exist with which to infer hidden system knowledge based on known inputs and outputs. The General Linear Model, for example, is useful because regression (equivalently least squares optimisation) provides optimal estimates of the activity-related weights that are subsequently processed to form statistical parameter maps. Riera et al. propose a method based on linearisation for estimating the physiological state variables in their nonlinear BOLD signal model [2].

In this paper we propose the novel application of particle filtering to the state estimation of the nonlinear, stochastic BOLD signal model. Particle filtering is a sequential Monte Carlo method in which probability densities are represented by a population of point masses, propagated through time according to the evolving system dynamics [6. Applications of particle filters range from radar tracking [7] to image registration [8]. Unlike the linearisation approach [2], the particle filter maintains the nonlinearities present in the signal model, and is therefore an ideal candidate for application to fMR data analyses. We demonstrate this to be a robust and accurate method for estimation of the BOLD signal model.

\section{The BOLD Signal Model}

The balloon model of Buxton et al. 3], and subsequent windkessel formulation of Mandeville et al. 4, provide a mechanistic model of the dependence of the BOLD signal, $y$, on cerebral blood flow, $f$, cerebral blood volume, $v$, and deoxyhaemoglobin content, $q$. In this model, the venous compartment is considered to be a balloon-like structure, inflated by increased blood flow resulting from an increase in local neuronal activity. The blood flow increases more than the cerebral metabolic rate of oxygen, thus locally reducing the deoxyhaemoglobin content which in turn causes a slight increase in the local MR signal, creating the BOLD effect [1]. The balloon model equations are as follows (for a full derivation, see [15]):

$$
\begin{aligned}
y_{t} & =h\left(v_{t}, q_{t}\right) \\
h\left(v_{t}, q_{t}\right) & =V_{0}\left(k_{1}\left(1-q_{t}\right)+k_{2}\left(1-\frac{q_{t}}{v_{t}}\right)+k_{3}\left(1-v_{t}\right)\right) \\
\dot{v}_{t} & =\frac{1}{\tau_{0}}\left(f_{t}-v_{t}^{1 / \alpha}\right) \\
\dot{q}_{t} & =\frac{1}{\tau_{0}}\left(\frac{f_{t}\left(1-\left(1-E_{0}\right)^{1 / f_{t}}\right)}{E_{0}}-\frac{q_{t}}{v_{t}^{1-1 / \alpha}}\right)
\end{aligned}
$$

where $E_{0}$ is the resting oxygen extraction fraction, $V_{0}$ is the resting blood volume fraction, and $\left\{k_{1}=7 E_{0}, k_{2}=2, k_{3}=2 E_{0}-0.3\right\}$ are generally accepted coefficient values [5. The parameter $\tau_{0}$ is the mean transit time of the venous compartment, and $\alpha$ is the stiffness component.

The relationship between an input stimulus sequence, $u$, the flow-inducing signal, $s$, and the cerebral blood flow, $f$, is a topic of ongoing research and 
is difficult to verify experimentally [1]. Friston et al. [5] propose the following parsimonious model linking stimulus to cerebral blood flow:

$$
\begin{aligned}
& \dot{s}_{t}=\varepsilon u_{t}-\frac{1}{\tau_{s}} s_{t}-\frac{1}{\tau_{f}}\left(f_{t}-1\right) \\
& \dot{f}_{t}=s_{t}
\end{aligned}
$$

Here $\tau_{s}$ is the signal decay time constant, $\tau_{f}$ the autoregulatory time constant, and $\varepsilon$ the neuronal efficacy.

Equations (10)-(6) describe a system of ordinary differential equations that deterministically relate input, $u_{t}$, to output, $y_{t}$, via a set of state variables, $\mathbf{x}_{t}=\left[v_{t}, q_{t}, s_{t}, f_{t}\right]^{\prime}$. Riera et al. [2] recently extended the above model to include a noise process in the state dynamics,

$$
\mathrm{d} \mathbf{x}_{t}=F\left(\mathbf{x}_{t}, u_{t}\right) \mathrm{d} t+\mathbf{g} \mathrm{d} \omega_{t}
$$

and similarly for the observation process (11). $F(\cdot)$ encompasses the right-hand sides of Equations (3) - (6), $\mathbf{g}$ is a vector of weighting terms, and $\omega_{t}$ is a Wiener process. Let $\boldsymbol{\Theta}=\left\{\varepsilon, \tau_{s}, \tau_{f}, \tau_{0}, \alpha, E_{0}, V_{0}, \mathbf{g}, \sigma_{e}^{2}\right\}$ denote the complete set of BOLD signal parameters.

\section{Method}

We describe the acquisition methods for simulated and experimental fMR data (Sections 3.1 3.2), followed by details of state estimation by particle filtering (Section 3.3) and by the linearisation approach of [2] (Section 3.4). A joint parameter and state estimation algorithm is then outlined in Section 3.5 .

\subsection{Simulated Data}

Ground truth against which to test the state estimation algorithms is not attainable through experimental fMR data, for which reason we simulate the BOLD signal model. Simulation requires discretisation of the continuous-time state dynamics (7), achieved by applying the Euler-Maruyama method with sampling at time instants $t_{k}=k \Delta_{t}$ [9]. The resultant discrete-time dynamics,

$$
\mathbf{x}_{k+1}=\mathbf{x}_{k}+F\left(\mathbf{x}_{k}, u_{k}\right) \Delta_{t}+\mathbf{g} w_{k}, \quad w_{k} \sim N\left(0, \Delta_{t}\right)
$$

converge in the mean square sense to the continuous-time dynamics for $\Delta_{t} \rightarrow 0$.

The input stimulus sequence, $u_{k}$, is simulated by a binary sequence representing the on/off conditions of a block design, or Gaussian impulses of duration $200 \mathrm{~ms}$ for an event design [2].

The output BOLD signal (1) is observed every TR seconds, $t_{k^{\prime}}=k^{\prime} \mathrm{TR}$, where TR is the Response Time of the scanning session:

$$
y_{k^{\prime}}=h\left(v_{k^{\prime}}, q_{k^{\prime}}\right)+e_{k^{\prime}}, \quad e_{k^{\prime}} \sim N\left(0, \sigma_{e}^{2}\right)
$$

The noise process, $e_{k^{\prime}}$, accounts for measurement and instrumentation noise encountered when acquiring the signal. 


\subsection{Experimental Data}

Data was acquired from four consecutive fMRI scans of a single subject. In each scan, the subject performed two two-minute blocks of a repetitive left-handed finger tapping task. Blocks were interspaced by two minute periods of eyes-open rest.

During each scan 240 sixteen-slice brain volumes were acquired at a rate of $0.5 \mathrm{~Hz}$, using a $\mathrm{T} 2 *$-weighted gradient-echo, echo-planar-imaging sequence. Slice thickness was $6 \mathrm{~mm}$, and in-plane voxel resolution $3.2 \times 3.2 \mathrm{~mm}^{2}$. Activation maps were generated with the FSL software package1, which uses a General Linear Modeling approach to detect regions with significant responses during the tapping task. The experimental data was obtained by detrending then averaging across the four scans the responses of voxels from a region of interest in the right primary motor cortex, centered at the most strongly responding voxel, as depicted in Fig. 1].

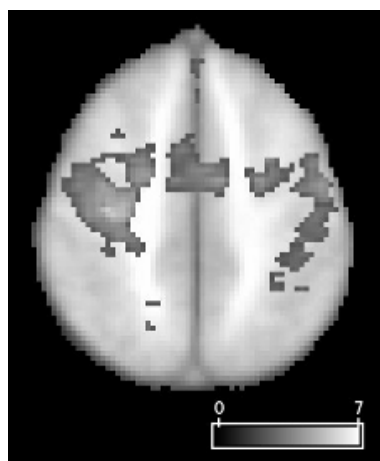

(a) Activation map

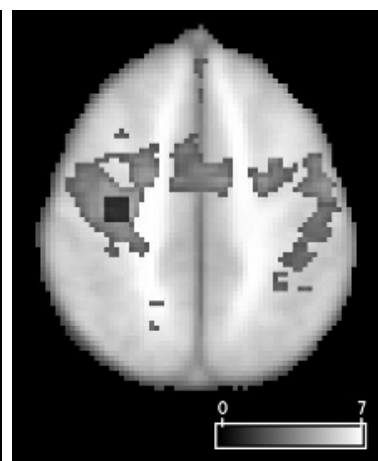

(b) ROI: black square

Fig. 1. A region of interest (ROI) is formed from a cluster of voxels in the right primary motor cortex, centred at the most strongly responding voxel

\subsection{State Estimation Via Particle Filtering}

Particle filters are a powerful class of techniques for implementing recursive Bayesian filters when the dynamics of the system under consideration are nonlinear and/or non-Gaussian [6]. The particle filter constructs the posterior density, $p\left(\mathbf{x}_{k} \mid y_{1}, \ldots, y_{k}\right)$, by a weighted sum of $N$ particles distributed over the support of $\mathbf{x}_{k}$ (refer to Algorithm 4, [6]):

$$
p\left(\mathbf{x}_{k} \mid y_{1}, \ldots, y_{k}, \boldsymbol{\Theta}\right)=\sum_{i=1}^{N} w_{k}^{i} \delta\left(\mathbf{x}_{k}-\mathbf{x}_{k}^{i}\right)
$$

The particles and weights are composed and propagated through time according to the following recursions:

\footnotetext{
${ }^{1}$ www.fmrib.ox.ac.uk/fsl/
} 


$$
x_{k}^{i} \sim p\left(\mathbf{x}_{k} \mid \mathbf{x}_{k-1}^{i}, \boldsymbol{\Theta}\right), \quad w_{k}^{i} \propto p\left(y_{k} \mid \mathbf{x}_{k}^{i}, \boldsymbol{\Theta}\right)
$$

State estimates, $\hat{X}=\left[\hat{\mathbf{x}}_{1}, \ldots, \hat{\mathbf{x}}_{T}\right]$, are formed by ergodic averages. The noise processes in the discrete-time state and observation equations (8)-(9) define the prior and likelihood densitites for use in (11):

$$
p\left(\mathbf{x}_{k} \mid \mathbf{x}_{k-1}\right) \sim N\left(F\left(\mathbf{x}_{k}, u_{k}\right), \mathbf{g g}^{\prime}\right), \quad p\left(y_{k} \mid \mathbf{x}_{k}\right) \sim N\left(h\left(\mathbf{x}_{k}\right), \sigma_{e}^{2}\right)
$$

\subsection{State Estimation Via Local Linearisation}

Linear functions, $F(\cdot, \cdot)$ and $h(\cdot)$, in (8) and (9) would render the Kalman filter the optimal state estimator, in the minimum variance sense [10]. No such optimal filter exists for the nonlinear dynamical system defining the hemodynamic response to a stimulus (8)-(9), hence the application of Monte Carlo methods such as the particle filter. An alternative method commonly employed to deal with system nonlinearity is to locally linearise the system nonlinearities. This is the approach taken in [2] to obtain estimates of the states $\mathbf{x}_{k}=\left[v_{k}, q_{k}, s_{k}, f_{k}\right]^{\prime}$, resulting in the linearised state equation,

$$
\begin{gathered}
\mathbf{x}_{k+1}=\mathbf{x}_{k}+\left[\Delta_{t} r_{0}\left(J_{f}^{x}\left(\mathbf{x}_{k}, u_{k}\right), \Delta_{t}\right)-r_{1}\left(J_{f}^{x}\left(\mathbf{x}_{k}, u_{k}\right), \Delta_{t}\right)\right] J_{f}^{t}\left(\mathbf{x}_{k}, u_{k}\right) \\
+r_{0}\left(J_{f}^{x}\left(\mathbf{x}_{k}, u_{k}\right), \Delta_{t}\right) F\left(\mathbf{x}_{k}, u_{k}\right)+\xi_{k}
\end{gathered}
$$

where $J_{f}^{x}$ is the Jacobian matrix, and $J_{f}^{t}$ the time derivative, of $F\left(\mathbf{x}_{t}, u_{t}\right)$, $r_{n}(M, a)=\int_{0}^{a} e^{M u} u^{n} \mathrm{~d} u$ and $\xi_{k}$ is a Gaussian noise process (see [2] for details). The linearisation filtering algorithm proceeds according to an extended Kalman filter type recursion through propagation of mean and covariance estimates [10] of linearised dynamics.

\subsection{Joint Parameter and State Estimation}

We employ an iterative coordinate descent approach [11 to simultaneously estimate the states and parameters of the BOLD signal model. At iteration $n$, given the current parameter estimates, $\boldsymbol{\Theta}^{(n-1)}$, the particle filter is applied to optimise the posterior,

$$
X^{(n)}=\arg \max _{X} p\left(X \mid y_{1}, \ldots, y_{T}, \mathbf{\Theta}^{(n-1)}\right)
$$

Updated parameter estimates at iteration $(n)$ are subsequently calculated by optimising the posterior, given the updated state sequence estimates, equivalently a maximum likelihood calculation:

$$
\boldsymbol{\Theta}^{(n)}=\arg \max _{\boldsymbol{\Theta}} p\left(X^{(n)} \mid y_{1}, \ldots, y_{T}, \boldsymbol{\Theta}\right)
$$

The optimisation (15) is carried out using MATLAB's fminsearch function. The iterative algorithm terminates when a maximum number of iterations is exceeded, or estimates are deemed to have converged. 


\section{Results}

\subsection{Simulated Data}

We demonstrate the accuracy and robustness of the particle filter through application to two sets of simulated fMR data, the first being an event response, the second a block design.
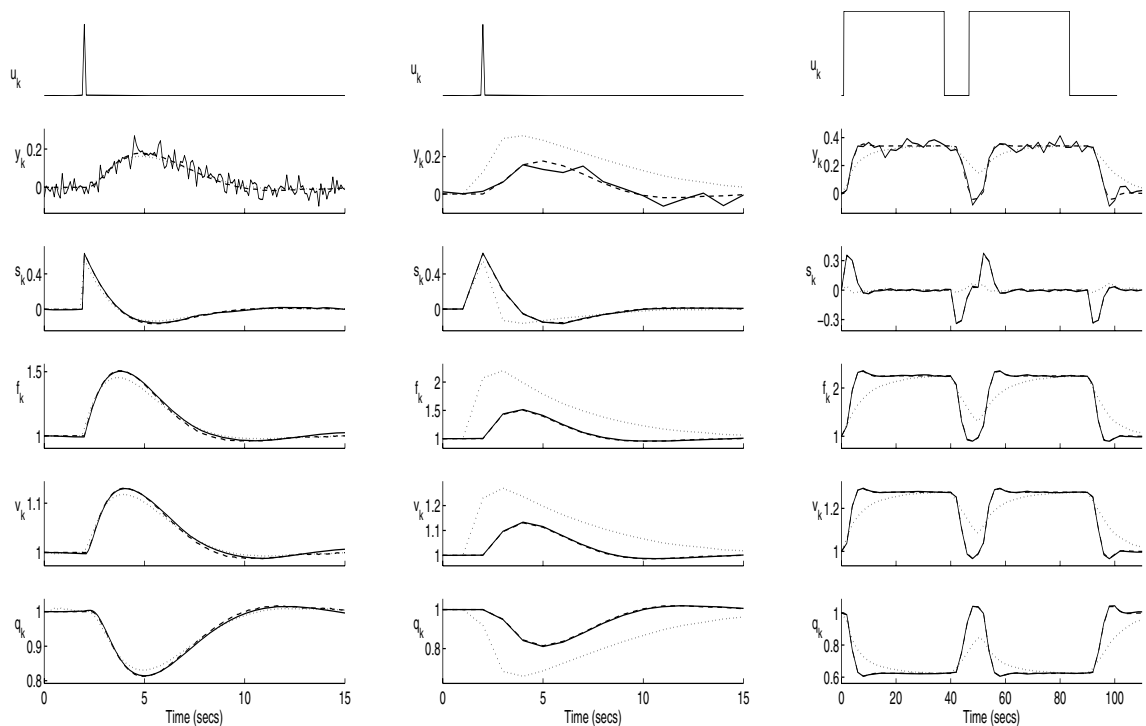

(a) Event design, $\mathrm{TR}=0.1$ (b) Event design, $\mathrm{TR}=1$ (c) Block design, $\mathrm{TR}=1 \mathrm{sec}$. sec. sec.

Fig. 2. State estimation of BOLD signal model. Axes (top to bottom): $u_{k}, y_{k}, s_{k}, f_{k}, v_{k}, q_{k}$. Solid line: True state/signal. Dashed line: Particle filter estimate. Dotted line: Linearisation filter estimate. Where not visible, the particle filter coincides with the true state/signal (solid line).

Consider the BOLD signal response to a single stimulus event, $u_{t}$, at $t=2$ seconds. The system parameters are chosen to be in their typical ranges [5]: $\varepsilon=0.5, \tau_{s}=1.25, \tau_{f}=2.5, \tau_{0}=1, \alpha=0.3, E_{0}=0.3$, with output scaling factor, $V_{0}=0.2$. The observation noise variance is $\sigma_{e}^{2}=10^{-3}$, and $\mathbf{g}=[0.01,0,0,0]^{\prime}$. The discrete-time state equation (8) is sampled at $\Delta_{t}=0.1$ seconds, while the observation sampling rate, $\mathrm{TR}$, is varied. Fig. $2 \mathrm{a}$ ) shows that for $\mathrm{TR}=\Delta_{t}=0.1$ seconds, the particle filter is more accurate than the linearisation algorithm. This difference is only exemplified as the TR increases to a more realistic sampling interval of TR $=1$ second, as shown in Fig. 2b). The particle filter maintains accuracy state estimates while the linearisation filter fails to track the state sequences.

Fig. 2r) depicts the results of the particle filtering and linearisation algorithms for a block design simulation. The system parameters are the same as for the 
event response. Again, the particle filter demonstrates superior performance to the linearisation method, and is able to track the changes in the hidden state variable sequences.

\subsection{Experimental Data}

The observed signal from the right primary motor cortex of a subject during a block-design finger-tapping task (see Sec. 3.2), and the experiment paradigm are shown in the top two axes of Fig. 3a). We apply the joint state and parameter estimation algorithm detailed in Section 3.5 to this input/output pair of sequences. Fig. 3a) displays the resultant state sequence estimates, Fig. 3b) the parameter estimate histories. All parameters converge within a few iterations and were found to be robust to reasonable change in initial conditions. To simplify computation, the parameters of the coupling between input stimulus and the cerebral blood flow (5)-(6) are assumed known; $\varepsilon=0.5, \tau_{s}=1.25$ and $\tau_{f}=2.5$, with no detriment to the estimation procedure.

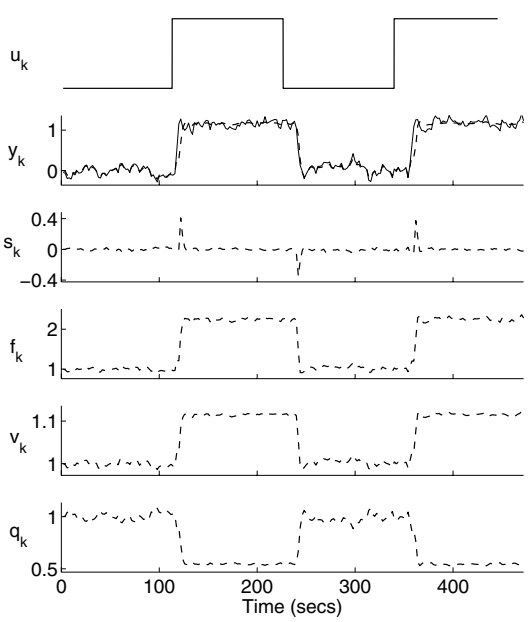

(a) State Estimates. Solid line: Observed signal. Dashed line: Particle filter estimate.
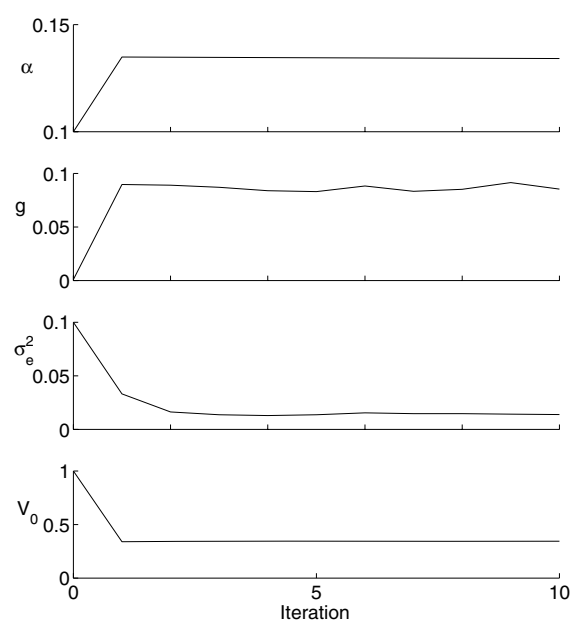

(b) Parameter Estimates.

Fig. 3. Results of the joint parameter and state estimation algorithm (Sec. 3.5) applied to observed right primary motor cortex signal during finger-tapping task

\section{Discussion and Conclusions}

The pathway from input stimulus sequence to output BOLD effect signal via modulation of physiological variables including deoxyhemoglobin content, cerebral blood flow and cerebral blood volume, is both nonlinear and stochastic. Our proposed particle filtering approach is therefore ideally suited to the task, given 
its inherent ability to maintain signal nonlinearity and propagate uncertainty. Superior performance of the particle filtering approach over the linearisation method has been demonstrated, while the proposed joint state and parameter estimation algorithm is successful in application to experimental fMR data. It is expected that nonlinear filtering methods, such as the particle filtering algorithm proposed in the paper, will become increasingly important in fMR analyses as limitations of linear and linearised modelling are reached.

\section{References}

1. Buxton, R.B., Uludağ, K., Dubowitz, D.J., Liu, T.T.: Modeling the hemodynamic response to brain activation. NeuroImage 23 (2004) S220-S233

2. Riera, J.J., Watanabe, J., Kazuki, I., Naoki, M., Aubert, E., Ozaki, T., Kawashima, R.: A state-space model of the hemodynamic approach: nonlinear filtering of BOLD signals. NeuroImage 21 (2004) 547-567

3. Buxton, R.B., Wong, E.C., Frank, L.R.: Dynamics of blood flow and oxygenation changes during brain activation: the Balloon model. Magnetic Resonance in Medicine 39 (1998) 855-864

4. Mandeville, J.B., Marota, J.J., Ayata, C., Zararchuk, G., Moskowitz, M.A., Rosen, B., Weisskoff, R.M.: Evidence of a cerebrovascular postarteriole windkessel with delayed compliance. Journal of Cerebral Blood Flow and Metabolism 19 (1999) 679-689

5. Friston, K.J., Mechelli, A., Turner, R., Price, C.J.: Nonlinear responses in fMRI: The Balloon model, Volterra kernels, and other hemodynamics. NeuroImage 12 (2000) 466-477

6. Arulampalam, M.S., Maskell, S., Gordon, N., Clapp, T.: A tutorial on particle filters for online nonlinear/non-Gaussian Bayesian tracking. IEEE Transactions on Signal Processing 50(2) (2002) 174-188

7. Gilks, W.R., Berzuini, C.: Following a moving target - Monte Carlo inference for dynamic Bayesian models. Journal of the Royal Statistical Society, Series B 63(1) (2001) 127-146

8. Ma, B., Ellis, R.E.: Unified point selection and surface-based registration using a particle filter. In: Proceedings of MICCAI 2005. (2005) 75-82

9. Gard, T.C.: Introduction to Stochastic Differential Equations. Marcel Dekker (1988)

10. Anderson, B.D.O., Moore, J.B.: Optimal Filtering. Prentice-Hall (1979)

11. Luenberger, D.G.: Linear and Nonlinear Programming. 2nd edn. Addison-Wesley (1984) 